\title{
Effects of pre-surgery physiotherapy on walking ability and lower extremity strength in patients with degenerative lumbar spine disorder: Secondary outcomes of the PREPARE randomised controlled trial
}

\author{
Maria Fors ${ }^{1,2^{*}}$ (D, Paul Enthoven ${ }^{1}$, Allan Abbott ${ }^{1}$ and Birgitta Öberg ${ }^{1}$
}

\begin{abstract}
Background: Degenerative lumbar spine disorders are common among musculoskeletal disorders. When disabling pain and radiculopathy persists after adequate course of rehabilitation and imaging confirms compressive pathology, surgical decompression is indicated. Prehabilitation aiming to augment functional capacity pre-surgery may improve physical function and activity levels pre and post-surgery. This study aims to evaluate the effect and dose-response of pre-surgery physiotherapy on quadriceps femoris strength and walking ability in patients with degenerative lumbar spine disorders compared to waiting-list controls and their association with postoperative physical activity level.
\end{abstract}

Method: In this single blinded, 2-arm randomised controlled trial, 197 patients were consecutively recruited. Inclusion criteria were: MRI confirmed diagnosis and scheduled for surgery due to disc herniation, lumbar spinal stenosis, degenerative disc disease or spondylolisthesis, ages 25-80 years. Patients were randomised to 9 weeks of pre-surgery physiotherapy or to waiting-list. Patient reported physical activity level, walking ability according to Oswestry Disability Index item 4, walking distance according to the SWESPINE national register and physical outcome measures including the timed ten-meter walk test, maximum voluntary isometric quadriceps femoris muscle strength, patient-rated were collected at baseline and follow-up. Parametric or non-parametric within and between group comparisons as well as multivariate regression was performed.

(Continued on next page)

\footnotetext{
* Correspondence: maria.fors@liu.se; https://www.imh.liu.se/fysioterapi

${ }^{1}$ Department of Medical and Health Sciences, Division of Physiotherapy,

Faculty of Medicine and Health Sciences, Linköping University, SE-581 83 Linköping, Sweden

${ }^{2}$ Department of Activity and Health, and Department of Medical and Health

Sciences, Linköping University, Linköping, Sweden
}

(c) The Author(s). 2019 Open Access This article is distributed under the terms of the Creative Commons Attribution 4.0 International License (http://creativecommons.org/licenses/by/4.0/), which permits unrestricted use, distribution, and reproduction in any medium, provided you give appropriate credit to the original author(s) and the source, provide a link to the Creative Commons license, and indicate if changes were made. The Creative Commons Public Domain Dedication waiver (http://creativecommons.org/publicdomain/zero/1.0/) applies to the data made available in this article, unless otherwise stated. 
(Continued from previous page)

Results: Patients who received pre-surgery physiotherapy significantly improved in all variables from baseline to follow-up $(p<0.001-p<0.05)$ and in comparison to waiting-list controls $(p<0.001-p<0.028)$. Patients adhering to $\geq 12$ treatment sessions significantly improved in all variables $(p<0.001-p<0.032)$ and those receiving $0-11$ treatment session in only normal walking speed (p0.035) but there were no significant differences when comparing dosages. Physical outcome measures after pre-surgery physiotherapy together significantly explain $27.5 \%$ of the variation in physical activity level 1 year after surgery with pre-surgery physical activity level having a significant multivariate association.

Conclusion: Pre-surgery physiotherapy increased walking ability and lower extremity strength in patients with degenerative lumbar spine disorders compared to waiting-list controls. A clear treatment dose-response response relationship was not found. These results implicate that pre-surgery physiotherapy can influence functional capacity before surgical treatment and has moderate associations with maintained postoperative physical activity levels mostly explained by physical activity level pre-surgery.

Trial registration: NCT02454400. Trial registration date: August 31st 2015, retrospectively registered.

Keywords: Degenerative lumbar spine disorder, Low Back pain, Physiotherapy, Rehabilitation, Exercise, Walking ability, Strength

\section{Background}

Low back pain is a common musculoskeletal disorder that has become a public health problem. The lifetime prevalence is estimated to $39 \%$ [1]. Although only $10 \%$ of low back pain disorders are diagnosed as lumbar spinal stenosis (LSS) or disc herniation, they are the most common cause for spinal surgery, followed by degenerative disc disease (DDD) and spondylolisthesis [2, $3]$. In the Swedish Spine register of spinal surgery (SWESPINE), the diagnosis LSS, disc herniation, spondylolisthesis and DDD are termed as degenerative lumbar spine disorders [3].

Degenerative lumbar spine disorders may cause pain and sensorimotor deficits in the lower extremities, affecting physical and self-rated extremity function [4, 5]. Impaired walking ability with regards to distance, speed and pattern is often seen in these patients [6-9]. Symptom-related activity restriction may also occur with implications for overall health and increased risk of comorbidities related to inactivity $[9,10]$. Limited walking ability is one of the main reasons of patients with LSS to seek care $[5,10,11]$ and a progressive reduction of walking distance, often due to leg pain, is according to some studies an indication for back surgery $[12,13]$.

Spinal surgery as treatment for degenerative lumbar spine disorders has over the last decade increased excessively in Sweden and the rest of the world [3, 14]. Only $10 \%$ of the studies on the effect of surgery report about non-surgical intervention prior to surgery for degenerative lumbar spine disorders [15]. However, it is standard practice in Sweden that an adequate period of primary care non-surgical treatment is trialed before decision making as recommended in the literature [16-20]. Prehabilitation on the other hand is aimed at augmenting functional capacity in patients selected for surgery and in that way possibly improve post-surgical outcomes [21, 22]. It is unknown how aspects of prehabilitation dosage may lead to these potential effects. In line with evidencebased guidelines for low back pain [23] and degenerative lumbar spine disorders [16-18], the effectiveness of structured physiotherapy in the prehabilitation context for surgical candidates has been investigated in the PREPARE trial $[24,25]$. It was shown the pre-surgery physiotherapy decreased pain, risk of avoidance behavior and worsening of psychological well-being, and improved quality of life more than surgical waiting-list controls in the pre-surgical phase. Patient reported physical activity was significantly higher after pre-surgery and was maintained one year after surgery compared to the waiting-list controls [25].

Despite that one of the primary goals with treatments for degenerative lumbar spine disorders is to improve pain related disability, especially walking ability, only few studies investigating treatment for this patient group provide thorough evaluation with not only patientreported outcomes but also objective physical assessments $[9,14,26]$. It is suggested that objective measures of function can complement self-rated measures to provide a comprehensive picture of patient disability [26]. However, a better understanding of how these measures are associated is required. In the PREPARE trial [24], objective physical assessments such as gait speed and lower extremity strength as well as patient-reported walking ability were collected as secondary outcomes during the prehabilitation phase conducted in the primary care setting. This to investigate if pre-surgery physiotherapy can augment pre-surgery physical capacity. Only selfreported measures could be performed post-surgery due to logistical reasons. The purpose of this report is therefore to investigate effectiveness of pre-surgery 
physiotherapy compared to waiting-list controls in the PREPARE trial [24] on walking ability and quadriceps femoris strength in patients with degenerative lumbar spine disorders following the intervention before surgery. An additional purpose is to investigate if differences exist between these physical outcomes based on the level of dosage adherence in prehabilitation and if pre-surgical physical measures have multivariate associations with self-reported physical activity level one year after surgery.

\section{Method}

\section{Study design}

This study is a single blinded, 2-arm, randomised controlled trial. The protocol has been published [24] and the patient reported core outcome set has been reported [25]. This paper presents secondary outcomes of muscle strength and walking ability. The CONSORT guidelines were followed for reporting [27]. The study was approved by the Regional Ethics committee (dnr 2012/16731). Patients' gave their written consent to participate in the study.

\section{Subjects and setting}

Patients were consecutively recruited at the Spinal Clinic at the University Hospital in Linköping Sweden between October 2012 and March 2015. All patients who were referred to the Spine Clinic were examined by an orthopedic spine surgeon. Inclusion criteria were: age of 2580 years, MRI confirmed diagnosis of disc herniation, LSS, DDD or spondylolisthesis (at least grade 4), scheduled for surgery, fluent in Swedish. Exclusion criteria were: indication for acute surgery, presence of severe spinal pathology, previous surgery on the same lumbar spinal level. A total of 242 patients met the inclusion criteria.

\section{Intervention}

Patients in the waiting-list group received usual care which included information about the surgical procedure, postoperative rehabilitation and advice regarding continued physical activity. Patients in the physiotherapy group received the same usual care intervention and the pre-surgery physiotherapy one-hour session twice a week for 9 weeks containing:

1. Active physiotherapy according to a treatmentbased classification (TBC) [28];

a. Specific exercise and mobilization, or b) Motor control exercises, or c) Traction. Treatment approach dependent upon assessment findings.

2. Tailor-made general exercise program performed in a gym supervised by a physiotherapist in one-onone sessions. The program included strength-, cardiovascular- and mobility exercises. Dose and intensity of the exercise were set and progressed over time. The program was also individualized to the patients' specific impairments.

3. Behavioral approach to increase activity level and decrease fear-avoidance behavior.

4. Daily physical activity for at least $30 \mathrm{~min} /$ day. The patient wrote a daily logbook over physical activity.

The interventions were performed at one of eleven physiotherapy public health care clinics in Östergötland County. The physiotherapists who delivered the intervention were trained and received directives for the treatment by two specialist physiotherapists. For each patient the physiotherapist followed a checklist with treatment and progression for each treatment-session. Modification of the treatment could be individually tailored. The intervention is further described in the study protocol by Lindbäck et al. [24].

\section{Outcome}

Physical outcome measures, including patient-reported outcome measures and objective outcome measures, were collected at baseline and after 9 weeks intervention pre-surgery.

Walking ability was measured through gait speed and self-rated questions regarding walking ability. To assess walking speed a $5-10 \mathrm{~m}$ distance is recommended in a wide range of populations $[29,30]$. Gait speed in meters/ second, was measured through a timed ten- meter walk test (10MWT). Patients walked $10 \mathrm{~m}$ on a straight path with $3 \mathrm{~m}$ for acceleration before and $3 \mathrm{~m}$ for deceleration after. Patients were asked to walk in their normal and fastest gait speed. Each pace was measured once. The $10 \mathrm{MWT}$ is reported to be a reliable and valid measurement for gait speed [31-33].

Self-rated walking ability was measured using item four of the Oswestry Disability Index (ODI) regarding how pain affects walking distance [34, 35]. There is evidence supporting its validity and reproducibility [36]. A question from SWESPINE, was used to evaluate selfrated walking ability. The question was: "How far can you walk at normal walk speed?" There were four answer alternatives: 1 . Less than $100 \mathrm{~m}$. 2. 100-500 m. 3 . $0.5 \mathrm{~km}-1 \mathrm{~km}$. 4. More than $1 \mathrm{~km}$. Physical activity level was measured after pre-surgery intervention and one year after surgery by a question with 5 answer options ranging from very little physical activity to regular strenuous physical activity.

Strength in the lower extremities was tested in the quadriceps femoris muscle. Maximum voluntary isometric muscle force was measured with a dynamometer model Chatillon CSD 500 strength dynamometer (Ametek, Largo, FL, USA). The dynamometer is sensitive to 
small changes in muscle strength [37] with good reliability [37-39]. The same measurement procedure was used as in former studies measuring normative values of maximum voluntary isometric force using a dynamometer $[40,41]$. The test was repeated two times on the right and on the left leg. If the second test score was higher than the first, a third test was done. The highest peak torque obtained was recorded in kilogram (Kg).

\section{Randomisation}

Block randomisation was used with sealed envelopes prepared for each randomisation block. An independent physiotherapist at the Spine Clinic performed the randomisation and informed the patient about group allocation.

\section{Blinding}

The two physiotherapists who performed the measurements at baseline and follow up were blinded to the randomization. The patient and the treating physiotherapist could not be blinded for test condition.

\section{Statistical analysis}

IBM SPSS $^{\bullet}$ version 25 was used in performance of statistical analyses. Variables were tested for normality using Shapiro-Wilks test and skewness. For comparison of data at baseline and follow-up between physiotherapyand waiting-list groups, Mann-Whitney U test was used for variables not normally distributed or categorical and Student's t-test was used for continuous and normally distributed variables. For within group comparisons paired Student's t-test or a Wilcoxon Signed-Rank test were used. A $P$-value of $<0.05$ was considered as statistically significant. Cohen $d$ effect size was calculated for change in group over time, where $d \geq 0.20$ was considered a small, $d \geq 0.50$ a medium and $d \geq 0.80$ was a large effect size [42]. Hedges $g$ was used to calculate effect size when the sample sizes where dissimilar [43]. Bivariate and multivariate associations between number of presurgery treatment sessions and physical outcome measures were analysed to investigate possible linear or nonlinear associations with regards to dose-response curves. Stepwise linear regression analysis was used to evaluate the multivariate associations between pre-surgical physical outcome measures (independent variables $=$ walking speed, Quadriceps strength, self-rated walking ability through ODI item 4 and a question from SWESPINE regarding walking distance) and physical activity level one year after surgery. In the first step, pre-surgery physical outcome measures were entered and as a second step pre-surgery physical activity level. The data assumptions required for linear regression modelling were test and confirmed.
Per protocol analyses was performed on data provided from patients' adherent to at least 12 treatment sessions of the intervention. The trial's a-priori sample size calculation was based on the ODI total score which was the primary outcome in the PREPARE Randomised controlled trial [24].

\section{Results}

One hundred ninety-seven patients with degenerative lumbar spine disorders were allocated to either waitinglist group $(n=98)$ or physiotherapy group (PT) $(n=99)$. A total of 171 patients were included in the per protocol analysis (Fig. 1).

No significant differences in the patients' characteristics were found between the two groups at baseline (Table 1).

The analyses revealed that the PT-group had a statistically significant improvement in gait speeds and in quadriceps femoris strength at follow-up directly after the 9 week pre-surgery intervention and in comparison to the waiting-list group. In the waiting-list group there were no significant changes in gait speeds between baseline and after 9 week pre-surgery intervention. There was a deterioration in maximum voluntary muscle force in both legs after intervention compared to baseline in the waiting-list group, with a right leg decrease in strength reaching statistical significance. There were no statistically significant differences between groups at baseline (Table 2).

The PT-group improved significantly in the ODI item four and the walking distance question from baseline to follow-up after pre-surgery intervention and in comparison to the waiting-list group (Table 3). There were no significant between group differences at baseline (ODI item four p 0.607, walking distance question p 0.558).

In a first step screening bivariate or multivariate associations (linear or non-linear) between number of presurgery treatment sessions and physical outcome measure showed no significant associations in regard to doseresponse curves. Therefore, a further analysis investigating differences in a dichotomized variable for pre-surgery treatment sessions $(0-11 / \geq 12$ sessions) was investigated. Patients who adhered to 12 or more treatment sessions showed statistically significant improvement in normal gait speed and fast gait speed and in maximum voluntary muscle force in the right and left Quadriceps. The patients who adhered to 0-11 treatment sessions had only a statistically significant improvement in normal gait speed. There were no statistically significant differences between groups at baseline and no differences in change over time between groups (Table 4).

The patients who adhered to 12 or more treatment sessions had a statistically significant improvement in ODI item four and self-rated walking distance. The patients who adhered to 0-11 treatment sessions did not 


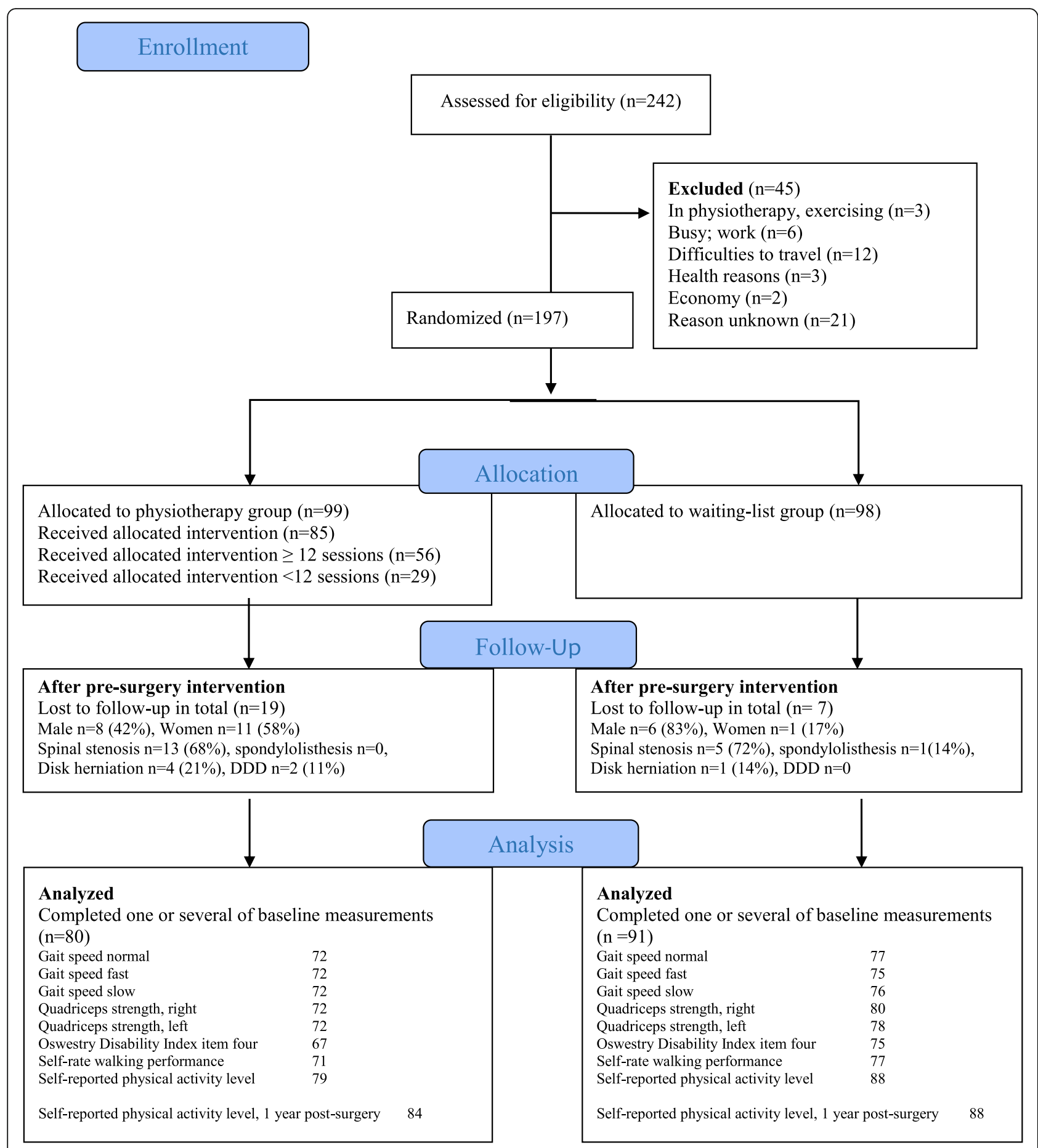

Fig. 1 Consolidated Standards of Reporting Trials (CONSORT) flowchart diagram of the randomised controlled trial

improve significantly (Table 5). There were no significant between group differences at baseline (ODI item four $\mathrm{p}$ 0.082, walking distance question $\mathrm{p}$ 0.346) or in change over time (ODI item four p 0.866, walking distance question p 0.542).

Pre-surgical gait speed, self-rated walking ability and Quadriceps strength together significantly explain
$17.4 \%(p=0.003)$ of variation in self-reported physical activity level one year after surgery in step 1. Adding pre-surgery physical activity level increased the explanatory value to $27.5 \%(\mathrm{p}=<0.001)$ in step 2 . In step 1 no single physical outcome measure alone was significant but in step 2, pre-surgery physical activity level alone had a significant association with physical 
Table 1 Baseline characteristics for all recruited patients and patients who completed one or several outcome measures at followup. Presented in mean and standard deviation or frequency. Significance level for differences in between groups

\begin{tabular}{|c|c|c|c|c|c|c|}
\hline & \multicolumn{3}{|l|}{ All recruited patients } & \multicolumn{3}{|l|}{ Analysed patient } \\
\hline & $\overline{\mathrm{PT}}(n=99)$ & Waiting-list $(n=98)$ & $P$-value & $\mathrm{PT}\left(n=80^{*}\right)$ & Waiting-list $\left(n=91^{*}\right)$ & $P$-value \\
\hline Gender, men (\%) & $45(45)$ & $47(49)$ & 0.725 & $37(46)$ & $45(50)$ & 0.573 \\
\hline Age, years & $57.9 \pm 13.3$ & $61 \pm 11.5$ & 0.082 & $59.2 \pm 12.5$ & $60.9 \pm 11.2$ & 0.349 \\
\hline Duration of current pain episode in months & $25.2 \pm 2.7(n=98)$ & $27.8 \pm 3.9(n=97)$ & 0.594 & $28.3 \pm 3$ & $29.3 \pm 4.1$ & 0.202 \\
\hline Duration in months & $304.6 \pm 29.7(n=80)$ & $289.2 \pm 29.3(n=87)$ & 0.738 & $307.9 \pm 29.6(n=64)$ & $277.4 \pm 28.6(n=81)$ & 0.531 \\
\hline Diagnosis (\%) & & & & & & 0.409 \\
\hline Spinal stenosis & $59(60)$ & $70(71)$ & & $52(65)$ & $66(72)$ & \\
\hline Disc herniation & $23(23)$ & $17(17)$ & & $13(16)$ & $15(17)$ & \\
\hline Spondylolisthesis & $8(8)$ & $7(7)$ & & $7(9)$ & $6(7)$ & \\
\hline DDD & $9(9)$ & $4(4)$ & 0.286 & $8(10)$ & $4(4)$ & \\
\hline \multicolumn{7}{|l|}{ Pain (Visual Analog Scale) } \\
\hline Back pain & $55.4 \pm 26.7(n=89)$ & $58.7 \pm 23.9(n=92)$ & 0.514 & $54.5 \pm 26.8(n=74)$ & $58.2 \pm 23.6(n=85)$ & 0.363 \\
\hline Leg pain & $65.4 \pm 23.8(n=88)$ & $64.8 \pm 21.5(n=91)$ & 0.620 & $63.8 \pm 24.5(n=74)$ & $63.8 \pm 21.7(n=84)$ & 0.983 \\
\hline
\end{tabular}

Bold text $\boldsymbol{p}<.05$

* Number of patients (n) in PT/Waiting-list group: Gait speed normal 72/77, Gait speed fast 72/75, Gait speed slow 72/76. Quadriceps Peak right 72/80, Quadriceps Peak left 72/78, ODI question four 67/75, Self-rate walking performance 71/77

activity level one year post-surgery $(\beta=0.281 p<$ 0.001) (Table 6).

\section{Discussion}

Walking ability is an important outcome as it correlates with several function- and health outcomes [32, 44-46] and is reported to be predictive for spine surgical outcomes $[7,47,48]$. The use of objective and self-reported walking measures as secondary outcomes in the PREPARE study [24], allows to capture a broader view of walking ability.

The result of the present study showed a significant improvement in gait speed, self-rated walking ability and in maximum voluntary isometric force in quadriceps femoris after 9 weeks pre-surgery physiotherapy interventions compared to waiting-list controls prior to surgery. The effect sizes were small but the effects might still be of importance in this group of patients with a quite high mean age. According to previous literature a change in gait speed of $0.10-0.17 \mathrm{~m} / \mathrm{s}$ is said to be a minimal clinically important difference across multiple patient groups [49]. The improvement in normal gait speed for the PT-group was similar to these results while change in fast gait speed surpassed this level both for within group and between group change. Thus, the study results indicate that patients with degenerative lumbar spine disorders with large physical impairment and who are candidates for surgical treatment still

Table 2 Means in gait speed (m/s) at normal and fast speed and in maximum voluntary muscle force (Kg) of the right and left m. Quadriceps at baseline and after 9 week pre-surgery intervention. Within- and between groups differences over time

\begin{tabular}{|c|c|c|c|c|c|c|c|c|}
\hline & \multirow[b]{2}{*}{$\mathrm{n}$} & \multirow{2}{*}{$\begin{array}{l}\text { Baseline } \\
\text { Mean (SD) }\end{array}$} & \multirow[b]{2}{*}{$p$-Value } & \multicolumn{2}{|l|}{ Within group changes } & \multirow[b]{2}{*}{ Effect size* } & \multicolumn{2}{|l|}{ Between group change } \\
\hline & & & & Mean (SD) change from baseline & $p$-Value & & Mean difference $(95 \% \mathrm{Cl})$ & $p$-Value \\
\hline \multicolumn{9}{|c|}{ Gait speed, normal } \\
\hline PT & 72 & $1.10(0.24)$ & 0.112 & $-0.09(0.14)$ & $<0.001$ & -0.34 & 0.07 (0.02 to 0.12$)$ & 0.005 \\
\hline Waiting-list & 77 & $1.13(0.24)$ & & $-0.01(0.17)$ & 0.556 & -0.04 & & \\
\hline \multicolumn{9}{|c|}{ Gait speed, fast } \\
\hline PT & 72 & $1.52(0.44)$ & 0.989 & $-0.12(0.22)$ & 0.001 & -0.29 & $0.21(0.07$ to 0.26$)$ & $<0.001$ \\
\hline Waiting-list & 75 & $1.53(0.38)$ & & $0.05(0.36)$ & 0.222 & 0.12 & & \\
\hline \multicolumn{9}{|c|}{ Quadriceps strength, right } \\
\hline PT & 72 & $22.20(8.08)$ & 0.958 & $-1.74(5.64)$ & 0.011 & -0.11 & $2.80(0.74$ to 4.87$)$ & $<0.001$ \\
\hline Waiting-list & 80 & $22.22(9.70)$ & & $1.54(6.51)$ & 0.026 & 0.16 & & \\
\hline \multicolumn{9}{|c|}{ Quadriceps strength, left } \\
\hline PT & 72 & $21.81(8.62)$ & 0.912 & $-2.40(7.31)$ & 0.006 & -0.25 & $3.28(1.30$ to 5.20$)$ & 0.003 \\
\hline Waiting-list & 78 & $21.80(9.10)$ & & $0.38(5.40)$ & 0.368 & 0.01 & & \\
\hline
\end{tabular}

PT, physiotherapy group; SD, standard deviation; $\mathrm{Cl}$, confidence interval. Bold text $p<. \mathbf{0 5}$. ${ }^{*}$ Cohen $d$ 
Table 3 Self-rated walking ability measured with Oswestry Disability Index (ODI) item four and self-rated walking distance at baseline and after 9 week pre-surgery intervention and between group changes. Changes within groups presented in n (\%)

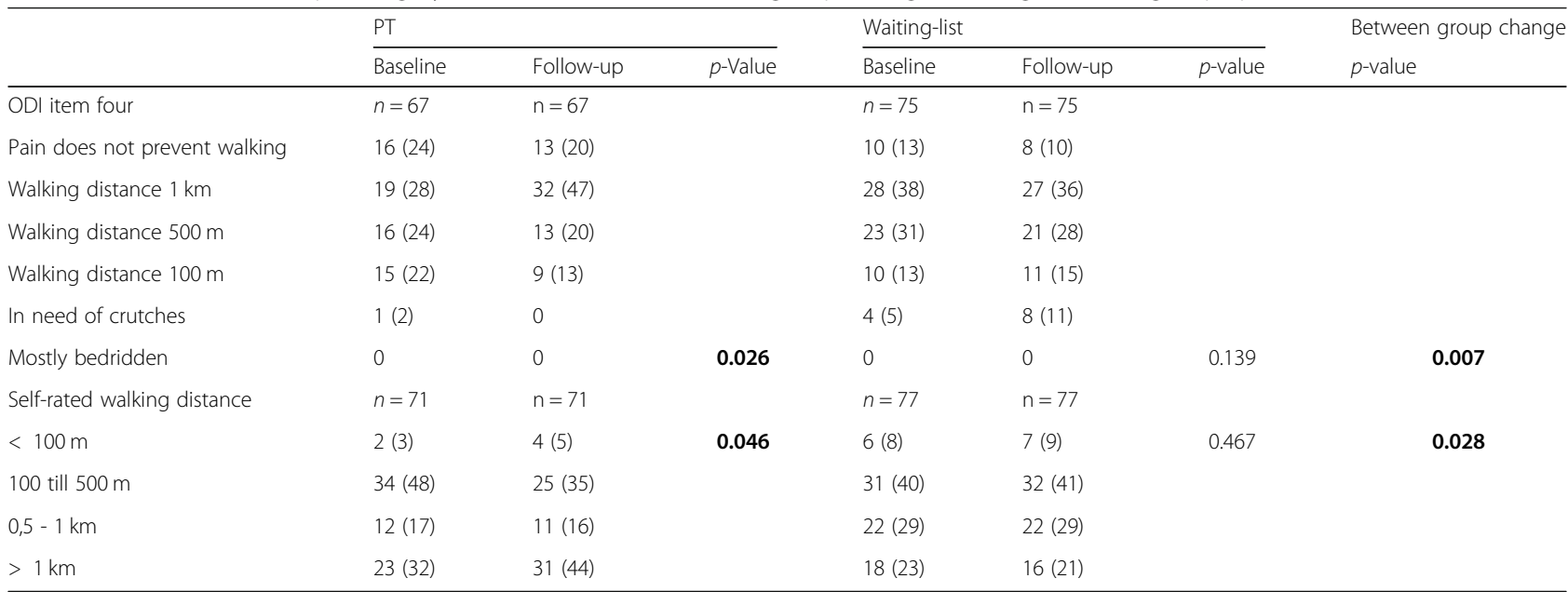

Bold text $p<.05$

have the potential to improve their physical function and benefit from physiotherapy. This suggests that there is a potential to improve physical function before surgery. The secondary analysis of the PREPARE study supports the previously reported effects on self-reported outcomes [25].

The current study is one of few studies including physical function outcomes with both self-rated and objective measurements. The results are in line with previous studies evaluating physical function in these patient groups in a prehabilitation phase [25, 50]. Only Nielsen et al. [50] have evaluated prehabiliation intervention in patients with degenerative lumbar spine disorders with physical performance functional tests. Unlike the results in the present study, Nielsen et al. [50] reported that only the self-

Table 4 Means in the PT-group gait speed $(\mathrm{m} / \mathrm{s})$ at normal and fast speed and in maximum voluntary muscle force $(\mathrm{Kg})$ of the right and left $\mathrm{m}$. Quadriceps at baseline and after 9 week pre-surgery intervention divided in number of treatment sessions. Within- and between groups differences over time

\begin{tabular}{|c|c|c|c|c|c|c|c|c|c|}
\hline & \multirow{2}{*}{$\begin{array}{l}\text { Patients } \\
\text { n, } \\
\text { Treatment } \\
\text { sessions } \\
\text { (median, } \\
\text { range) }\end{array}$} & \multirow{2}{*}{$\begin{array}{l}\text { Baseline } \\
\text { Mean (SD) }\end{array}$} & \multirow[b]{2}{*}{$\begin{array}{l}p- \\
\text { Value }\end{array}$} & \multicolumn{2}{|c|}{ Within group changes } & \multirow[b]{2}{*}{$\begin{array}{l}\text { Effect } \\
\text { size }^{*}\end{array}$} & \multicolumn{2}{|l|}{ Between group change } & \multirow[b]{2}{*}{ Effect size ${ }^{* *}(95 \% \mathrm{Cl})$} \\
\hline & & & & $\begin{array}{l}\text { Mean (SD) } \\
\text { change } \\
\text { from baseline }\end{array}$ & $p$-Value & & $\begin{array}{l}\text { Mean difference }(95 \% \\
\text { Cl) }\end{array}$ & $\begin{array}{l}p- \\
\text { Value }\end{array}$ & \\
\hline \multicolumn{10}{|l|}{ Gait speed normal } \\
\hline 0-11 treatment sessions & $17(5,0-10)$ & $1.15(0.14)$ & 0.711 & $-0.06(0.10)$ & 0.035 & -0.37 & $0.04(-0.04$ to 0.12$)$ & 0.305 & $-0.312(-0.635-0.012)$ \\
\hline $\begin{array}{l}\geq 12 \text { treatment } \\
\text { sessions }\end{array}$ & $55(18,12-23)$ & $1.12(0.27)$ & & $-0.10(0.15)$ & $\begin{array}{l}< \\
0.001\end{array}$ & -0.37 & & & \\
\hline \multicolumn{10}{|l|}{ Gait speed fast } \\
\hline $0-11$ treatment sessions & $17(5,0-10)$ & $1.54(0.32)$ & 0.857 & $-0.05(0.22)$ & 0.299 & -0.21 & $0.08(-0.04$ to 0.19$)$ & 0.181 & $-0.372(-0.698--0.046)$ \\
\hline $\begin{array}{l}\geq 12 \text { treatment } \\
\text { sessions }\end{array}$ & $55(18,12-23)$ & $1.52(0.40)$ & & $-0.13(0.21)$ & $\begin{array}{l}< \\
0.001\end{array}$ & -0.84 & & & \\
\hline \multicolumn{10}{|l|}{ Quadriceps strength, right } \\
\hline 0-11 treatment sessions & $18(5,0-10)$ & $\begin{array}{l}25.23 \\
(9.01)\end{array}$ & 0.069 & $-1.93(7.50)$ & 0.289 & -0.19 & $-0.26(-3.34$ to 2.80$)$ & 0.865 & $0.014(-0,277-0.36)$ \\
\hline $\begin{array}{l}\geq 12 \text { treatment } \\
\text { sessions }\end{array}$ & $54(18,12-23)$ & $\begin{array}{l}21.23 \\
(7.58)\end{array}$ & & $-1.67(4.96)$ & 0.016 & -0.20 & & & \\
\hline \multicolumn{10}{|l|}{ Quadriceps strength, left } \\
\hline $0-11$ treatment sessions & $18(5,0-10)$ & $\begin{array}{l}22.51 \\
(8.30)\end{array}$ & 0.765 & $-3.82(10.50)$ & 0.141 & -0.36 & $-1.90(-5.80$ to 2.10$)$ & 0.354 & $0.22(-0.101-0.541)$ \\
\hline $\begin{array}{l}\geq 12 \text { treatment } \\
\text { sessions }\end{array}$ & $54(18,12-23)$ & $\begin{array}{l}21.80 \\
(8.81)\end{array}$ & & $-1.96(5.96)$ & 0.019 & -0.22 & & & \\
\hline
\end{tabular}

$S D$ standard deviation, $\mathrm{Cl}$ confidence interval. Bold text $\mathrm{p}<. \mathbf{0 5}$. ${ }^{*}$ Cohen $d,{ }^{* *}$ Hedges $g$ 
Table 5 Self-rated walking ability measured with Oswestry Disability Index (ODI) item four and self-rated walking distance at baseline and after 9 week pre-surgery intervention divided in number of treatment sessions. Changes within groups over time

\begin{tabular}{|c|c|c|c|c|c|c|}
\hline & \multicolumn{3}{|c|}{$0-11$ treatment session } & \multicolumn{3}{|c|}{$\geq 12$ treatment session } \\
\hline & Baseline & Follow-up & $\overline{p \text {-Value }}$ & Baseline & Follow-up & $\overline{p \text {-Value }}$ \\
\hline \multicolumn{7}{|l|}{ ODI item four } \\
\hline Treatment sessions (median, range) & $n=18(5,0-10)$ & $\mathrm{n}=18(5,0-10)$ & 0.763 & $n=49(18,12-23)$ & $\mathrm{n}=49(18,12-23)$ & \\
\hline Pain does not prevent walking & $6(33)$ & $5(28)$ & & $10(20)$ & $8(16)$ & \\
\hline Walking distance $1 \mathrm{~km}$ & $7(39)$ & $8(44)$ & & $12(25)$ & $24(49)$ & \\
\hline Walking distance 500 m & $4(22)$ & $3(17)$ & & $12(25)$ & $10(21)$ & \\
\hline Walking distance 100 m & $1(6)$ & $2(11)$ & & $14(28)$ & $7(14)$ & \\
\hline In need of crutches & 0 & 0 & & $1(2)$ & 0 & \\
\hline Mostly bedridden & 0 & 0 & & 0 & 0 & 0.006 \\
\hline \multicolumn{7}{|l|}{ Self-rated walking distance } \\
\hline Treatment sessions (median, range) & $n=21(5,0-10)$ & $n=21(5,0-10)$ & 0.783 & $n=50(18,12-23)$ & $\mathrm{n}=50(18,12-23)$ & \\
\hline$<100 \mathrm{~m}$ & $1(5)$ & $1(5)$ & & $1(2)$ & $3(6)$ & 0.032 \\
\hline 100 till $500 \mathrm{~m}$ & $8(38)$ & $8(38)$ & & $26(52)$ & $17(34)$ & \\
\hline $0,5-1 \mathrm{~km}$ & $3(14)$ & $2(10)$ & & $9(18)$ & $9(18)$ & \\
\hline$>1 \mathrm{~km}$ & $9(43)$ & $10(47)$ & & $14(28)$ & $21(42)$ & \\
\hline
\end{tabular}

Bold text $p<.05$

rated physical function improved after pre-surgery intervention.

Numerous physiotherapy interventions have been recommended for patients with degenerative lumbar spine disorders $[14,17,19,20,51,52]$. The physiotherapeutic intervention tested in the present study is multidimensional and targets several aspects of the biopsychosocial model [53]. The intervention is based on a treatment-based classification, the exercise is tailormade and with a behavioral approach to meet the different demands in a heterogenous population. This does not allow to draw conclusions about single interventions. When considering a dose-response relationship, participation in $>12$ treatment sessions resulted in statistically significant improvements but not statistically significant compared with $<11$ treatment sessions concerning physical measures. When considering effect sizes, the larger change in fast gait speed after $>12$ treatment sessions and the interpretation of between group effect size confidence intervals $=-0.372(95 \% \mathrm{CI}-0.698--0.046)$ may support a potential for statistically significant difference between groups if a larger sample size was included in the study. The reasoning for analysing a cut-off of 12 treatment session can be supported by a previously published meta-regression analysis showing improvement of pain and function after similar interventions for degenerative musculoskeletal conditions such as osteoarthritis [54].

Table 6 Stepwise regression analysis estimating multivariate association between preoperative physical outcome measures and physical activity level (independent variables) and physical activity level one year after surgery (dependent variable) $(n=108)$

\begin{tabular}{|c|c|c|c|c|}
\hline \multirow[b]{2}{*}{ Independent variable } & \multicolumn{2}{|l|}{ Step 1} & \multicolumn{2}{|l|}{ Step 2} \\
\hline & Unstandardized Beta (SE) & $P$-value & Unstandardized Beta (SE) & $P$-value \\
\hline Gait speed, normal & $0.430(0.57)$ & 0.449 & $0.502(0.53)$ & 0.348 \\
\hline Gait speed, fast & $0.203(0.39)$ & 0.604 & $-0.159(0.38)$ & 0.677 \\
\hline Quadriceps strength, right & $0.021(0.02)$ & 0.325 & $0.020(0.02)$ & 0.330 \\
\hline Quadriceps strength, left & $-0.011(0.01)$ & 0.550 & $-0.010(0.01)$ & 0.547 \\
\hline ODI item four & $-0.123(0.12)$ & 0.309 & $-0.062(0.11)$ & 0.586 \\
\hline Self-rated walking distance & $0.079(0.13)$ & 0.548 & $0.084(0.12)$ & 0.499 \\
\hline \multirow[t]{2}{*}{ Physical activity level } & & & $0.281(0.07)$ & $<0.001$ \\
\hline & R Square $=0.174$ & 0.003 & R Square $=0.275$ & $<0.001$ \\
\hline
\end{tabular}


Previous results from the PREPARE study published by Lindbäck et al. [25] show an improvement in physical activity level and in fear-avoidance beliefs after the presurgery intervention. Fear avoidance behavior as an attempt to reduce pain is common amongst persons with degenerative lumbar disorders and low back pain [12, 55]. The results on the physical measures can therefore be influenced both by a real change in physical capacity and by behavioral components as for example fear avoidance. Studies indicates that interventions for low back pain that addressed fear-avoidance beliefs are more effective than interventions based on only biomedical concepts [55] and treatment including self-management and psychological approaches are recommended [56]. Nielsen et al. [50] reported that pre-surgery self-rated physical function enhanced in patients who executed a daily home exercise-program for two months. This compared to the supervised training in the physiotherapy clinic performed in the present study in order to enhance self-efficacy, reduce avoidance behavior and ensure progression and dose of the training. Considering these results, it is of interest to further examine what specific components and mechanisms in the physical therapy lead to a successful intervention.

The significant improvements in the PT-group and the significant differences between the groups indicate that this patient group with long standing pain problems and large physical impairments tolerate and can benefit from a structured rehabilitation period. Preoperative physical fitness, physical activity and self-rated health predicts better surgical outcome in general $[21,22,57]$ and after spinal surgery [47]. Therefore, preventing physical deterioration and making the patient better physically prepared for the surgical procedure and the following rehabilitation while waiting for surgery may lead to better post-surgery outcomes. Previous studies $[50,58]$ have not shown effects of pre-surgery intervention on long term postsurgical pain and physical function after lumbar surgery. Although they differ in intervention and study population compared to each other and to the PREPARE study [24]. Like previous literature [36-38] no side-effects from the physiotherapy intervention where reported. Lindbäck et al. [25] reports that only $58 \%$ of the patients in the PREPARE study had $\geq 1$ visit to a physiotherapist or other caregivers in the last 12 months before assessment for spinal surgery. It is important to follow the guideline recommendation to exhaust nonsurgical interventions before decision on surgery. It is also obvious that the knowledge concerning the role of physical function still is scarce and further development of pre-surgery interventions is needed.

The study results should be interpreted with consideration to the methodological strength and limitations of the study. The physiotherapy intervention was planned to meet the different needs in heterogenous population and therefore the tailored physiotherapeutic intervention by the use of TBC and tailored exercises can be considered to be a strength. It is important in studies of physiotherapy interventions that the interventions are performed according to the instructions. The supervised training, check-list and follow-up meetings aimed to ensure dosage and progression of the intervention. On the other hand, treatment was given at eleven physiotherapy clinics giving a risk for disparity.

The gait test used in the study fulfills the demands of using a valid clinical measure, reflecting a part of patients' walking capacity. It is associated with other physical functions for example aerobic capacity, muscle strength, postural control, and individuals' general health status $[29,30]$. It could be of interest to add further measures of walking distance as suggested by TomkinsLane et al. [59] and Ammendolia et al. [26]. Although, the results from objective and self-reported measures in present study are consistent and support the conclusion of effects on walking.

The inclusion of patients was dependent on the surgeons decision and they may not be representative for the entire spine surgeon community. Consensus is lacking regarding indications or guidelines for surgical treatment for this patient group [51,60].

There was a drop-out of $19 \%$ in the PT-group and 7\% in the waiting-list group.

The reason for drop-out were unknown. Although there were no specific patient groups amongst the dropout regarding gender or diagnosis.

Previous results from the PREPARE study [25] showed a significant higher self-reported physical activity level pre- and post-surgery in the PT-group. The current study showed that pre-surgical physical activity level, Quadriceps strength, walking speed and walking ability together have a moderate multivariate association with physical activity level one year after surgery. The only independent variable that had a significant association was pre-surgery level of physical activity. This highlights the importance of assessing patients' physical activity level and to focus on increasing or maintaining physical activity level as an important part in a pre-surgical intervention. It is of interest to further investigate the significance of pre-surgery physical measures and physical activity levels for the longitudinal outcome regarding physical activity level post-surgery.

In conclusion, the results of this secondary analysis on physical outcomes of the PREPARE study [24] showed that the multidimensional pre-surgery intervention had positive effects on walking ability and Quadriceps strength. Even with fairly small effect sizes, these effects might be of importance in a pre-surgery phase with risk of deterioration of physical capacity. The results confirm 
the previously reported outcomes on self-reported measures [25].

There is still however a need for further studies to analyse the most important components in a pre-surgery program and to study dose-effect relationships in exercises.

\section{Conclusion}

Pre-surgery physiotherapy increased walking ability and lower extremity strength in patients with degenerative lumbar spine disorders compared to waiting-list controls. A clear treatment dose-response response relationship was not found. These results implicate that presurgery physiotherapy can influence functional capacity before surgical treatment and has moderate associations with maintained postoperative physical activity levels mostly explained by physical activity level pre-surgery.

\section{Abbreviations}

10MWT: Ten-meters walk test; Cl: Confidence interval; DDD: Degenerative disc disease; LSS: Lumbar spinal stenosis; MRI: Magnetic resonance imaging; ODI: Oswestry disability index; PT: Physiotherapist; SD: Standard deviation; TBC: Treatment-based classification

\section{Acknowledgements \\ We thank Yvonne Lindbäck, RPT PhD, and Hans Tropp, MD PhD, as researchers active in designing and implementing the PREPARE study.}

\section{Consent for publications}

Not applicable.

\section{Authors' contributions}

BÖ was responsible for the design and planning of the study. All authors were involved in the preparation and planning of the data analyses. MF, AA and PE performed the statistical analyses. MF analysed and interpreted the patient data with supervision and participation from BÖ, AA and PE. MF did the drafting of the first manuscript with contributions from the other authors. All authors read and approved the final manuscript.

\section{Funding}

The study was partly funded by a Östergötland region public health care research grant. The funding body had no participation in the study's design, data collection, analyses and manuscript writing.

\section{Availability of data and materials}

The dataset used and/or analysed during the current study are available from the corresponding author on reasonable request.

\section{Ethics approval and consent to participate}

The PREPARE study was approved by the Regional Ethics Committee of the Faculty of Health sciences of Linköping university (dnr 2012/167-31).

Patients' gave their written consent to participate in the study.

\section{Competing interests}

AA is an associate editor at BMC Musculoskeletal Disorders. The authors have no competing interest to declare.

Received: 4 July 2019 Accepted: 23 September 2019

Published online: 24 October 2019

\section{References}

1. Hoy D, Williams G, March L, Brooks P, Blyth F, Woolf A, Vos TBR. A systematic review of the global prevalence of low back pain. Arthritis Rheum. 2012;64(6):2028-37.
2. Gibson J, Waddell G. Surgical interventions for lumbar disc prolapse. In: Gibson JA, editor. Cochrane Database of Systematic Reviews. Chichester, UK: John Wiley \& Sons, Ltd; 2007. p. CD001350.

3. Strömqvist B, Fritzell $P$, Hägg $O$, Jönsson B, Sandén B. Swedish Society of Spinal Surgeons. Swespine: the Swedish spine register : the 2012 report. Eur Spine J. 2013;22(4):953-74.

4. Winter CC, Brandes M, Müller C, Schubert T, Ringling M, Hillmann A, et al. Walking ability during daily life in patients with osteoarthritis of the knee or the hip and lumbar spinal stenosis: a cross sectional study. BMC Musculoskelet Disord. 2010;11(1):233.

5. Iversen MKJ. Research Report Examination Findings and Self-Reported Walking Capacity in Patients With Lumbar Spinal Stenosis. Phys Ther. 2001; 81:1296-306

6. Jönsson B, Strömqvist B. The straight leg raising test and the severity of symptoms in lumbar disc herniation. A preoperative evaluation, Spine (Phila Pa 1976). 1995 Jan 1;20(1):27-30.

7. Jansson KA, Nemeth G, Granath F, Jonsson B, Blomqvist P. Health-related quality of life in patients before and after surgery for a herniated lumbar disc. J Bone Joint Surg Br. 2005;87(7):959-64.

8. Suda Y, Saitou M, Shibasaki K, Yamazaki N, Chiba K, Toyama Y. Gait analysis of patients with neurogenic intermittent claudication. Spine (Phila Pa 1976). 2002;27(22):2509-13.

9. Tomkins-Lane CC, Holz SC, Yamakawa KS, Phalke W, Quint DJ, Miner J, et al. Predictors of walking performance and walking capacity in people with lumbar spinal stenosis, low back pain, and asymptomatic controls. Arch Phys Med Rehabil. 2012;93(4):647-53.

10. Norden J, Smuck M, Sinha A, Hu R, Tomkins-Lane C. Objective measurement of free-living physical activity (performance) in lumbar spinal stenosis: are physical activity guidelines being met? Spinal J. 2017;17:26-33.

11. Morag E, Hurwitz DE, Andriacchi TP, Hickey M, Andersson GB. Abnormalities in muscle function during gait in relation to the level of lumbar disc herniation. Spine (Phila Pa 1976). 2000;25(7):829-33.

12. Zeifang F, Schiltenwolf M, Abel R, Moradi B. Gait analysis does not correlate with clinical and MR imaging parameters in patients with symptomatic lumbar spinal stenosis. BMC Musculoskelet Disord. 2008;9(1):89-97.

13. Fritz JM, Lurie JD, Zhao W, Whitman JM, Delitto A, Brennan GP, et al. Associations between physical therapy and long-term outcomes for individuals with lumbar spinal stenosis in the SPORT study. Spine J. 2014; 14(8):1611-21.

14. Ammendolia C, Stuber K, Rok E, Rampersaud R, Kennedy CA, Pennick V, et al. Nonoperative treatment for lumbar spinal stenosis with neurogenic claudication. Ammendolia C, editor. Cochrane Database Syst Rev. 2013 Aug 30;(8):CD010712

15. Jacobs WCH, Rubinstein SM, Willems PC, Moojen WA, Pellisé F, Oner CF, et al. The evidence on surgical interventions for low back disorders, an overview of systematic reviews. Eur Spine J. 2013;22(9):1936-49.

16. Kreiner DS, Hwang SW, Easa JE, Resnick DK, Baisden JL, Bess S, et al. An evidence-based clinical guideline for the diagnosis and treatment of lumbar disc herniation with radiculopathy. Spine J. 2014;14(1):180-91.

17. Kreiner DS, Shaffer WO, Baisden JL, Gilbert TJ, Summers JT, Toton JF, et al. An evidence-based clinical guideline for the diagnosis and treatment of degenerative lumbar spinal stenosis (update). Spine J. 2013;13(7):734-43.

18. Watters WC 3rd, Bono CM, Gilbert TJ, Kreiner DS, Mazanec DJ, Shaffer WO, Baisden J, Easa JE, Fernand R, Ghiselli G, Heggeness MH, Mendel RC, O'Neill C, Reitman CA, Resnick DK, Summers JT, Timmons RBTJ. North American Spine Society an evidence-based clinical guideline for the diagnosis and treatment of degenerative lumbar spondylolisthesis. Spine J. 2009;9(7):609-14.

19. The National Institute for Health and Care Excellence (NICE). Low back pain and sciatica in over 16s: assessment and management. London; 2016.

20. Qaseem A, Wilt TJ, McLean RM, Forciea MA. Clinical Guidelines Committee of the American College of Physicians. Noninvasive Treatments for Acute, Subacute, and Chronic Low Back Pain: A Clinical Practice Guideline From the American College of Physicians. Ann Intern Med. 2017;166(7):514.

21. Santa Mina D, Clarke H, Ritvo P, Leung YW, Matthew AG, Katz J, et al. Effect of total-body prehabilitation on postoperative outcomes: a systematic review and meta-analysis. Physiother (United Kingdom). 2014; 100(3):196-207.

22. Carli F, Zavorsky GS. Optimizing functional exercise capacity in the elderly surgical population. Curr Opin Clin Nutr Metab Care. 2005;8(1):23-32. 
23. Delitto A, George SZ, Van Dillen L, Whitman JM, Sowa G, Shekelle P, Denninger TRGJ. Low Back pain clinical practice guidelines linked to the international classification of functioning, disability, and health from the Orthopaedic section of the American Physical Therapy Association. J Orthop Sport Phys Ther. 2012;42(4):A1-57.

24. Lindbäck Y, Tropp H, Enthoven P, Abbott A, Öberg B. PREPARE: pre-surgery physiotherapy for patients with degenerative lumbar spine disorder: a randomized controlled trial protocol. BMC Musculoskelet Disord. 2016;17(1):270.

25. Lindbäck Y, Tropp H, Enthoven P, Abbott A, Öberg B. Prepare: pre-surgery physiotherapy for patients with degenerative lumbar spine disorder: a randomized controlled trial. Spine J. 2018;18(8):1347-55.

26. Ammendolia C, Stuber K, Tomkins-Lane C, Schneider M, Rampersaud YR, Furlan AD, et al. What interventions improve walking ability in neurogenic claudication with lumbar spinal stenosis? A systematic review. Eur Spine J. 2014;23(6):1282-301.

27. Boutron I, Altman DG, Moher D, Schulz KF, Ravaud P. CONSORT statement for randomized trials of nonpharmacologic treatments: a 2017 update and a CONSORT extension for nonpharmacologic trial abstracts. Ann Intern Med. 2017;167(1):40

28. Stanton TR, Fritz JM, Hancock MJ, Latimer J, Maher CG, Wand BMPE. Evaluation of a treatment-based classification algorithm for low back pain: a cross-sectional study. Phys Ther. 2011;91(4):496-509.

29. Fritz S, Lusardi M. Walking speed: the sixth vital sign. J Geriatr Phys Ther. 2009:32(2):1-5

30. Middleton A, Fritz S, Lusardi M. Waking speed: the functional vital sign. J Aging Phys Act. 2015;23(2):314-22.

31. Steffen TM, Hacker T. A, Mollinger L. age- and gender-related test performance in community-dwelling elderly people: six-minute walk test, berg balance scale, timed up \&amp; go test, and gait speeds. Phys Ther. 2002;82(2):128-37.

32. Rydwik E, Bergland A, Forsen L, Frandin K. Investigation into the reliability and validity of the measurement of elderly people's clinical walking speed: a systematic review. Physiother Theory Pract. 2012;28(3):238-56.

33. Richards CL. Hemiparetic gait following stroke. Part 2: recovery and physical therapy. Gait Posture. 1996;4(2):149-62.

34. Fairbank JC, Couper JDJ. The Oswestry low back pain disability questionnaire. Physiotherapy. 1980;66:271-3.

35. Fairbank JC, Pynsent PB. The Oswestry disability index. Spine (Phila Pa 1976). 2000;25(22):2940-52.

36. Tomkins-Lane CC, Battié MC. Validity and reproducibility of self-report measures of walking capacity in lumbar spinal stenosis. Spine (Phila Pa 1976). 2010;35(23):2097-102.

37. Scott DA, Bond EQ, Sisto SA, Nadler SF. The intra- and interrater reliability of hip muscle strength assessments using a handheld versus a portable dynamometer anchoring station. Arch Phys Med Rehabil. 2004;85(4):598-603.

38. Mentiplay BF, Perraton LG, Bower KJ, Adair B, Pua Y-H, Williams GP, et al. Assessment of lower limb muscle strength and power using hand-Held and fixed dynamometry: a reliability and validity study. PLoS One. 2015;10(10):1-18.

39. Arnold CM, Warkentin KD, Chilibeck PD, Magnus CRA. The reliability and validity of handheld dynamometry for the measurement of lower-extremity muscle strength in older adults. J Strength Cond Res. 2010;24(3):815-24.

40. Andrews AW, Thomas MW, Bohannon RW. Normative values for isometric muscle force measurements obtained with hand-held dynamometers. Phys Ther. 1996;76(3):248-59.

41. Mangione KK, Craik RL, Tomlinson SS, Palombaro KM. Can elderly patients who have had a hip fracture perform moderate- to high-intensity exercise at home? Phys Ther. 2005;85(8):727-39.

42. Cohen J. A power primer. Psychol Bull. 1992;112(1):155-9.

43. Hedges LV. Distribution theory for Glass's estimator of effect size and related estimators. J Educ Stat. 1981;6(2):107-28.

44. Tomkins-Lane CC, Battié MC. Predictors of objectively measured walking capacity in people with degenerative lumbar spinal stenosis. J Back Musculoskelet Rehabil. 2013;26(4):345-52.

45. Cruz-Jimenez M. Normal changes in gait and mobility problems in the elderly. Phys Med Rehabil Clin N Am. 2017;28(4):713-25.

46. Cooper R, Kuh D, Hardy R. Mortality Review Group, FALCon and HALCyon Study Teams. Objectively measured physical capability levels and mortality: systematic review and meta-analysis. BMJ. 2010;341(9):c4467.

47. Aalto TJ, Malmivaara A, Kovacs F, Herno A, Alen M, Salmi L, et al. Preoperative predictors for postoperative clinical outcome in lumbar spinal stenosis. Spine (Phila Pa 1976). 2006;31(18):E648-63.
48. Sigmundsson FG, Kang XP, Jönsson B, Strömqvist B. Prognostic factors in lumbar spinal stenosis surgery. Acta Orthop. 2012;83(5):536-42.

49. Bohannon RW, Glenney SS. Minimal clinically important difference for change in comfortable gait speed of adults with pathology: a systematic review. J Eval Clin Pract. 2014;20(4):295-300.

50. Nielsen P, Jørgensen L, Dahl B, Pedersen T. Prehabilitation and early rehabilitation after spinal surgery: randomized clinical trial. Clin Rehabil. 2010;24:137-48

51. Zaina F, Tomkins-Lane C, Carragee E, Negrini S. Surgical versus non-surgical treatment for lumbar spinal stenosis. Cochrane Database Syst Rev. 2016;1: 857-68.

52. Watters WC, Baisden J, Gilbert TJ, Kreiner S, Resnick DK, Bono CM, et al. Degenerative lumbar spinal stenosis: an evidence-based clinical guideline for the diagnosis and treatment of degenerative lumbar spinal stenosis. Spine J. 2008:8(2):305-10.

53. Engel GL. The need for a new medical model: a challenge for biomedicine. Science (80- ). 1977;196(4286):129-36.

54. Juhl C, Christensen R, Roos EM, Zhang W, Lund H. Impact of exercise type and dose on pain and disability in knee osteoarthritis: a systematic review and meta-regression analysis of randomized controlled trials. Arthritis Rheumatol. 2014;66(3):622-36.

55. Wertli MM, Rasmussen-Barr E, Held U, Weiser S, Bachmann LM, Brunner F. Fear-avoidance beliefs-a moderator of treatment efficacy in patients with low back pain: a systematic review. Spine J. 2014;14(11):2658-78.

56. O'Connell NE, Cook CE, Wand BM, Ward SP. Clinical guidelines for low back pain: a critical review of consensus and inconsistencies across three major guidelines. Best Pract Res Clin Rheumatol. 2016;30(6):968-80.

57. Santa Mina D, Scheede-Bergdahl C, Gillis C, Carli F. Optimization of surgical outcomes with prehabilitation. Appl Physiol Nutr Metab. 2015;40(9):966-9.

58. Louw A, Diener I, Landers MR, Puentedura EJ. Preoperative pain neuroscience education for lumbar radiculopathy: a multi-center randomized controlled trial with one-year follow-up. Spine (Phila Pa 1976). 2014;39(18):1449-57.

59. Tomkins-Lane C, Battie M, Rogers T, Jiang H, Petersen S. A criterion measure of walking capacity in lumbar spinal stenosis and its comparison with a treadmill protocol. Spine (Phila Pa 1976). 2009;34(2444):9.

60. Debono B, Lonjon G, Galovich LA, Kerever S, Guiot B, Eicker S-O, Hamel O, Ringel F. Indication variability in degenerative-lumbar spine surgery: a 4nation survey. Spine (Phila Pa 1976). 2018;1(43):185-92.

\section{Publisher's Note}

Springer Nature remains neutral with regard to jurisdictional claims in published maps and institutional affiliations.

Ready to submit your research? Choose BMC and benefit from:

- fast, convenient online submission

- thorough peer review by experienced researchers in your field

- rapid publication on acceptance

- support for research data, including large and complex data types

- gold Open Access which fosters wider collaboration and increased citations

- maximum visibility for your research: over $100 \mathrm{M}$ website views per year

At $\mathrm{BMC}$, research is always in progress.

Learn more biomedcentral.com/submissions 